\title{
Descending Thoracic Aorta-abdominal Aortic Bypass and Bilateral Renal Arterial Blood Circulation Reconstruction Are Effective in Atypical Coarctation of the Aorta With Heart Failure: a Case Report
}

Takuma Mikami ( $\nabla$ t.mikami.02.28@gmail.com )

Sapporo Medical University: Sapporo Ika Daigaku https://orcid.org/0000-0002-4948-4005

Takeshi Kamada

Sapporo Medical University: Sapporo Ika Daigaku

Toshiyuki Yano

Sapporo Medical University: Sapporo Ika Daigaku

Tomohiro Nakajima

Sapporo Medical University: Sapporo Ika Daigaku

Naomi Yasuda

Sapporo Medical University: Sapporo Ika Daigaku

Tsuyoshi Shibata

Sapporo Medical University: Sapporo Ika Daigaku

Keitaro Nakanishi

Sapporo Medical University: Sapporo Ika Daigaku

Ryo Harada

Sapporo Medical University: Sapporo Ika Daigaku

Syuichi Naraoka

Sapporo Medical University: Sapporo Ika Daigaku

Kojiro Toda

Sapporo Medical University: Sapporo Ika Daigaku

Nobutaka Nagano

Sapporo Medical University: Sapporo Ika Daigaku

Atsuko Muranaka

Sapporo Medical University: Sapporo Ika Daigaku

Nobuyoshi Kawaharada

Sapporo Medical University: Sapporo Ika Daigaku

\section{Case report}


Keywords: atypical coarctation of the aorta, anatomical bypass, heart failure

Posted Date: June 28th, 2021

DOI: https://doi.org/10.21203/rs.3.rs-641932/v1

License: (c) (1) This work is licensed under a Creative Commons Attribution 4.0 International License. Read Full License

Version of Record: A version of this preprint was published at Journal of Cardiothoracic Surgery on August 4th, 2021. See the published version at https://doi.org/10.1186/s13019-021-01598-5. 


\section{Abstract}

Background: There are many reports on renal failure and heart failure due to coarctation of the aorta. However, there are no case reports in which revascularization dramatically improved left ventricular function in patients with progressive decline in left ventricular function. Herein, we present a rare case in which the left ventricular function dramatically improved after surgical treatment in a patient with progressive left ventricular dysfunction due to atypical coarctation of the aorta.

Case presentation: A 58-year-old man underwent axilobifemoral bypass at another hospital for atypical coarctation of the aorta due to Takayasu's arteritis. Approximately 10 years later, he was re-hospitalized for heart failure, and his left ventricular ejection fraction gradually decreased to $28 \%$. Computed tomography showed severe calcification and stenosis at the same site from the peripheral thoracic descending aorta to the lower abdominal aorta up to the renal arteries, and aortography showed delayed bilateral renal artery blood flow. An increase in plasma renin activity was also observed. Despite the administration of multiple antihypertensive drugs, blood pressure control was insufficient. We decided to perform surgical treatment to improve progressive cardiac dysfunction due to increased afterload and activated plasma renin activity. Descending thoracic aorta-abdominal aorta bypass and revascularization of both renal arteries via a great saphenous vein grafts were performed. Postoperative blood pressure control improved, and the dose of antihypertensive drugs could be reduced. Plasma renin activity decreased, and transthoracic echocardiography performed 1.5 years later showed improvement in contractility with a left ventricular ejection fraction of $58 \%$.

Conclusion: In patients with atypical coarctation of the aorta and decreased bilateral renal blood flow, heart failure due to renal hypertension, and progressive decrease in left ventricular contractility, descending thoracic aorta-abdominal aortic bypass and bilateral renal artery recirculation can be extremely effective.

\section{Background}

Some reports have shown that axillary-bilateral femoral bypass is effective in cases of hypertensive heart failure due to atypical coarctation of the aorta [1,2]. Axillofemoral bypass has been reported to have good long-term results [3]; however, there are also reports of poor long-term results, such as graft occlusion [4]. To the best of our knowledge, there are no reported cases that required re-bypass for atypical coarctation of the aorta in the long-term period after axillobifemoral bypass. Furthermore, although atypical coarctation of the aorta combined with hypertensive heart failure has been reported [1], there are no reports of cases that required bilateral renal artery reconstruction using open surgery or improvement of decreased left ventricular contractility. Herein, we report a case of aortic stenosis due to atypical coarctation of the aorta in a patient who had undergone axillobifemoral bypass and repeated hypertensive heart failure postoperatively. He underwent open surgery for descending thoracic aortaabdominal aorta bypass and bilateral renal artery reconstruction. After surgery, the left ventricular contractility improved significantly. 


\section{Case Presentation}

A 58-year-old man previously underwent axillobifemoral bypass at another hospital for atypical coarctation of the aorta due to Takayasu's arteritis. He was re-hospitalized approximately 10 years later due to heart failure, and a progressive decrease in left ventricular contractility was observed, along with a decrease in the left ventricular ejection fraction (LVEF) from 54-28\%. Multiple antihypertensive drugs were administered, and he was referred to our hospital to investigate the cause of the progressive decrease in left ventricular contractility and repeated heart failure due to poor blood pressure control. ${ }^{18} \mathrm{~F}$ fluorodeoxyglucose $\left({ }^{18} \mathrm{~F}-\mathrm{FDG}\right)$ positron emission tomography/computed tomography (PET/CT) performed at the previous hospital did not detect ${ }^{18} \mathrm{~F}-\mathrm{FDG}$ accumulation in the aorta. His laboratory data were as follows: creatinine, $2.93 \mathrm{mg} / \mathrm{dL}$; blood urea nitrogen, $65.7 \mathrm{mg} / \mathrm{dL}$; estimated glomerular filtration rate, $19 \mathrm{~mL} / \mathrm{min} / 1.73 \mathrm{~m}^{2}$; C-reactive protein (CRP), $0.54 \mathrm{mg} / \mathrm{dL}$; N-terminal pro-brain natriuretic peptide (NT-proBNP), 17,014 pg/mL; aldosterone, 119 (reference values 36-240) pg/ml; and plasma renin activity, 10.6 (reference values $0.2-2.3) \mathrm{ng} / \mathrm{ml} / \mathrm{h}$. His blood pressure values were as follows: right upper limb, 168/108 mmHg; upper left limb, 169/99 mmHg; right lower limb, 143/98 mmHg; and left lower limb, $131 / 101 \mathrm{mmHg}$. The right and left ankle brachial index (ABI) values were 0.85 and 0.78 , respectively. Preoperative transthoracic echocardiography revealed a LVEF of $28 \%$. There was no left ventricular asynergy, and the wall motion showed severe diffuse hypokinesis. Left ventricular wall thickness of the interventricular septum (IVS) and posterior wall (PW) were 13 and $12 \mathrm{~mm}$, respectively. Left ventricular hypertrophy was also observed. Further, the left ventricular end-diastolic and systolic diameters (LVEDD and LVESD) were 57.4 and $47.5 \mathrm{~mm}$, respectively, and left ventricular hypertrophy was detected. However, no significant valvular dysfunction was observed. Contrast-enhanced CT showed a patent axillobifemoral bypass and collateral circulation to the arteries of the lower extremity (Fig. 1a). Significant calcification was observed from the distal descending thoracic aorta to the abdominal aorta, and severe stenosis was suspected at the same site (Fig. 1b). Aortography showed severe stenosis of the aorta from below the celiac artery to the abdominal aorta below the renal arteries. We also noticed that collateral circulation from the celiac artery to the superior mesenteric artery was well developed Blood flow in the superior mesenteric artery passed from the developed collateral circulation from the celiac artery, and flow imaging of both renal arteries was delayed (Fig. 2a). Coronary angiography showed no abnormal findings, including at the entrance. Contrast-enhanced cardiac magnetic resonance imaging (MRI) revealed no apparent delayed contrast on the left ventricular wall, and T1 mapping images of cardiac MRI presented no significant increase in the T1 value on the left ventricular wall $(1,050 \mathrm{~ms}$ by $1.5 \mathrm{~T}$ modified Look-Locker inversion recovery method; the average value of normal control at our hospital was 1,000 $\mathrm{ms}$ ), and fibrosis of the left ventricular wall was considered mild (Fig. 2b). Although CRP was weakly positive, CT only showed severe calcification of the aorta, and PET-CT performed at the previous hospital did not show active inflammation of the aorta due to Takayasu's arteritis. Therefore, we decided to perform open surgery for uncontrolled renal hypertension and a progressive decrease in left ventricular contractility due to decreased bilateral renal blood flow. To reliably reduce afterload, we performed descending thoracic aorta-abdominal aorta bypass using a large-diameter graft and revascularization of both renal arteries via great saphenous vein grafts. 
Thoracotomy was performed under general anesthesia via the left lower 7th intercostal space, and the retroperitoneal approach was used to access the abdominal aorta. A skin incision was then made across the axillobifemoral bypass. To secure blood flow to the lower limbs during aortic clamping, surgery was performed without transection of the axillobifemoral bypass graft. The thoracic and abdominal aorta approach was performed without incising the diaphragm. In the retroperitoneal space, the dorsal side of the kidney was dissected, and the diaphragm was incised on the left side of the aortic hiatus to create a tunnel that connected the left thoracic cavity and retroperitoneal space. After systemic heparinization, the descending thoracic aorta was clamped at the Th9 level, and a proximal anastomosis was performed using a 12-mm woven graft (Intergard $\circledast$, Maquet, Sunderland, UK). The anastomosed woven graft was guided into the retroperitoneal space through the tunnel in the diaphragm, and a distal anastomosis was performed just above the bifurcation of the abdominal aorta. During this period, blood flow to the lower limb was maintained by the patent left axilobifemoral artery bypass. The anterior side of the left kidney was peeled off to expose the anterior surface of the abdominal aorta and the left and right renal arteries. The great saphenous vein was collected and anastomosed to the left and right renal arteries, and the vein graft stump was anastomosed to the woven graft. Next, the left leg of the exposed axillobifemoral bypass graft was resected just below the wound to reduce the risk of postoperative infection. The operative time was 388 minutes.

No postoperative complications were observed. Postoperative 3D-CT showed that the bypass graft from the descending thoracic aorta to the abdomen ran parallel to the aorta on the dorsal side of the left renal artery and was patent. The great saphenous vein graft to both renal arteries was also patent (Fig. 2c). Postoperative $\mathrm{ABI}$ improved to 1.12 on the right and 1.06 on the left; the upper-limb blood pressure improved to $128 / 91 \mathrm{mmHg}$ with good control, and the dose of antihypertensive drugs could be reduced. He was discharged from the hospital 16 days postoperatively. Blood test findings after discharge showed a decrease in NT-proBNP level $(78 \mathrm{pg} / \mathrm{dL})$, aldosterone level $(58 \mathrm{pg} / \mathrm{mL})$, and plasma renin activity $(1.1$ $\mathrm{ng} / \mathrm{ml} / \mathrm{h}$ ). Transthoracic echocardiography performed 1.5 years postoperatively showed marked improvement in LVEF of $58 \%$ (Fig. 3). Furthermore, left ventricular wall thickness and left ventricular diameter decreased: IVS, $9.0 \mathrm{~mm}$; PW, $8.7 \mathrm{~mm}$; LVEDD, $41.6 \mathrm{~mm}$; and LVESD, $28.6 \mathrm{~mm}$. Left ventricular reverse remodeling had also occurred.

\section{Discussion And Conclusions}

Atypical coarctation of the aorta is defined as stenosis outside the aortic isthmus, in contrast to typical coarctation of the aorta, which is characterized by stenosis of the aortic isthmus. The causes of atypical coarctation of the aorta include inflammatory vascular lesions (mainly Takayasu's arteritis), arteriosclerosis, and congenital anomalies [5]. Hypertension in the upper body due to decreased renal blood flow and increased vascular resistance causes increased left ventricular afterload and severe complications, resulting in a poor prognosis [6].

In the patient in this study, an axillary-bilateral femoral bypass had been performed approximately 10 years prior to rehospitalization; although the bypass was very patent, uncontrolled upper-limb 
hypertension and heart failure due to increased left ventricular afterload were observed. The left ventricular contractility decreased progressively. In addition, plasma renin activity was high, and both renal arteries showed delayed contrast enhancement on aortic angiography, suggesting a decrease in bilateral renal blood flow. Open surgery was indicated because few findings were suggestive of active Takayasu's arteritis. Surgical treatment methods, such as graft replacement, patch formation, bypass surgery, and stent placement [7], have been reported. Among these, the route of bypass has been frequently discussed, and many reports have included aorto-aortic bypass or axillary-femoral bypass. Some case reports have presented cases of axillary-femoral bypass that did not require thoracotomy or laparotomy and was effective as well as minimally invasive [1, 2]. However, reducing the afterload may be insufficient because it is bypassed by a small-diameter graft and because perfusion of abdominal organ now passed through retrograde blood flow from the femoral artery. Poor long-term patency rate of grafts was also reported [4]. Aorto-aortic bypass can be used with large-diameter grafts, leading to reliable reduction of left ventricular afterload, and revascularization of abdominal branches is possible depending on the route. However, its drawback is the high level of surgical invasiveness because it involves thoracotomy and laparotomy. In addition, either the ascending aorta or descending aorta will be selected as the inflow of the bypass graft. There are various reports on the route and method [8-10], each with specific advantages and disadvantages. When the ascending aorta is selected as the graft inflow, it is necessary to use a side clamp when anastomosing the ascending aorta or clamping the ascending aorta under cardiac arrest with the establishment of the cardiopulmonary bypass. In either case, the burden on the heart is massive, especially in cases of severe cardiac dysfunction. In addition, the graft may become long and kinked, and some grafts may pass through the abdominal cavity, resulting in digestive tract complications. When the descending aorta is selected as the graft inflow, the graft length is shortened, and it is possible to revascularize the abdominal branch from the retroperitoneum, and this is an advantage. However, aortic surgery with left thoracotomy requires separate lung ventilation, which is difficult in patients with severe respiratory dysfunction, and increases the risk of postoperative respiratory complications [11]. In this case, a reliable reduction in left ventricular afterload and improvement in bilateral renal artery perfusion are necessary. Therefore, surgery was selected for aorto-aortic bypass with the left thoracotomy retroperitoneal approach and revascularization of the bilateral renal arteries using a great saphenous vein graft. Simultaneous visual field of the bypassed graft and bilateral renal arteries is required for revascularization of the bilateral renal arteries. Therefore, during aorto-aortic bypassing, a tunnel was created through the aortic hiatus via the retroperitoneal approach from the dorsal side of the left kidney, and the ventral side of the left kidney was peeled off. It was possible to secure a simultaneous visual field and reconstruct both renal arteries.

Renal dysfunction and cardiac dysfunction are closely related, and the interaction between them is known as cardiorenal syndrome [12]. Hypertension and decreased renal blood flow are known to induce an increase in the renin-angiotensin-aldosterone (RAA) system and other specialized pathways. Fibrosis of the myocardium and kidneys have been reported to cause chronic and irreversible deterioration of cardiac and renal functions [13]. In this case, the plasma renin activity was predicted to increase, so the RAA system was enhanced due to the decrease in renal blood flow accompanying the progression of the 
coarctation of the aorta, and the increase in left ventricular afterload led to a progressive decrease in left ventricular contractility. Preoperative T1 mapping images by cardiac MRI showed a slight increase in the T1 value of the left ventricular wall. Therefore, fibrosis of the left ventricular wall was mild, and reverse remodeling of the left ventricle postoperatively and improvement of left ventricular contractility could be expected. Reduction of afterload and improvement of RAA system activation due to the thoracic descending aorta-abdominal aorta bypass and reconstruction of both renal arteries led to an improvement in left ventricular function.

In conclusion, we encountered a rare case of decreased bilateral renal blood flow and heart failure due to atypical coarctation of the aorta and progressive decrease in left ventricular contractility. Improvement in cardiac function was observed after thoracic descending aorta-abdominal aortic bypass and reconstruction of both renal arteries.

\section{Abbreviations}

LVEF: left ventricular ejection fraction

${ }^{18}$ F-FDG: ${ }^{18}$ F-fluorodeoxyglucose

PET-CT: positron emission tomography/computed tomography

CRP: C-reactive protein

NT-proBNP: N-terminal pro-brain natriuretic peptide

ABI: ankle brachial index

IVS: interventricular septum

PW: posterior wall

LVEDD: left ventricular end-diastolic diameter

LVESD: left ventricular end-systolic diameter

MRI: magnetic resonance imaging

RAA: renin-angiotensin-aldosterone

\section{Declarations}

\section{Ethics approval and consent to participate}

This study was conducted with approval from the Ethics Committee of The Sapporo Medical University of Medicine. 


\section{Consent for publication}

Written consent for publication is obtained from the patient.

\section{Availability of data and materials}

The datasets used and/or analyzed during the current study are available from the corresponding author on reasonable request.

\section{Competing interests}

The authors declare that they have no competing interests.

\section{Funding}

Not applicable.

\section{Authors' contributions}

$\mathrm{TM}, \mathrm{TK}, \mathrm{TY}$, and $\mathrm{N}$ collected the primary data and drafted the initial manuscript. $\mathrm{RH}, \mathrm{KT}, \mathrm{NN}$, and $\mathrm{AM}$ diagnosed the case and instructed the patient's treatment. TN, NY, TS, KN, SN assisted with the collection of the patient's data. All authors contributed to discussions and critically appraised the manuscript. All authors read and approved the final manuscript.

\section{Acknowledgements}

No applicable

\section{References}

1. Ishizuka M, Yamada S, Maemura S, Yamamoto K, Takizawa M, Uozumi H, et al. Axillofemoral bypass markedly improved acute decompensated heart failure and kidney injury in a patient with severely calcified stenosis of thoracoabdominal aorta (atypical aortic coarctation). Int Heart J. 2017;58:8203.

2. Fukunaga $\mathrm{N}$, Uryuhara $\mathrm{K}$, Koyama T. Axillobifemoral bypass for aortitis syndrome in a living-donor liver transplant patient. Ann Vasc Dis. 2016;9:114-6. d

3. Samson RH, Showalter DP, Lepore MR Jr, Nair DG, Dorsay DA, Morales RE. Improved patency after axillofemoral bypass for aortoiliac occlusive disease. J Vasc Surg. 2018;68:1430-7.

4. Schneider JR, McDaniel MD, Walsh DB, Zwolak RM, Cronenwett JL. Axillofemoral bypass: outcome and hemodynamic results in high-risk patients. J Vasc Surg. 1992;15:952-62.

5. Celoria GC, Patton RB. Congenital absence of aortic arch. Am Heart J. 1959;58:407-13.

6. Taketani T, Miyata T, Morota T, Takamoto S. Surgical treatment of atypical aortic coarctation complicating Takayasu's arteritis-experience with 33 cases over 44 years. J Vasc Surg. 2005;41:597- 
601.

7. D.S. Keith, B. Markey, M. Schiedler. Successful long-term stenting of an atypical descending aortic coarctation. J Vasc Surg. 2002;35:166-7.

8. Obata S, Mukai S, Morimoto H, Hiraoka T, Uchida H, Yamane Y. Successful ascending aortaabdominal aorta bypass graft through the left thoracic cavity in a patient with atypical coarctation. Ann Vasc Dis. 2013;6:670-3.

9. Terada T, Yuasa T, Hasegawa M, Horiuchi K, Nakata S, Yasuura K. Ascending to abdominal aorta extraanatomic bypass for descending aortic coarctation: a reconstruction technique without laparotomy or left thoracotomy. Ann Vasc Dis. 2014;7:354-8.

10. Kim YS, Cho YH, Sung K, Kim DK, Chung S, Park TK, et al. Clinical outcome of extraanatomic bypass for midaortic syndrome caused by Takayasu Arteritis. Ann Thorac Surg. 2020;109:1419-25.

11. Etz CD, Zoli S, Kari FA, Mueller CS, Bodian CA, Di Luozzo G, et al. Redo lateral thoracotomy for reoperative descending and thoracoabdominal aortic repair: a consecutive series of 60 patients. Ann Thorac Surg. 2009;88:758-66.

12. Zannad F, Rossignol P. Cardiorenal syndrome revisited. Circulation. 2018;138:929-44.

13. Rockey DC, Bell PD, Hill JA. Fibrosis-a common pathway to organ injury and failure. N Engl J Med. 2015;372:1138-49.

\section{Figures}




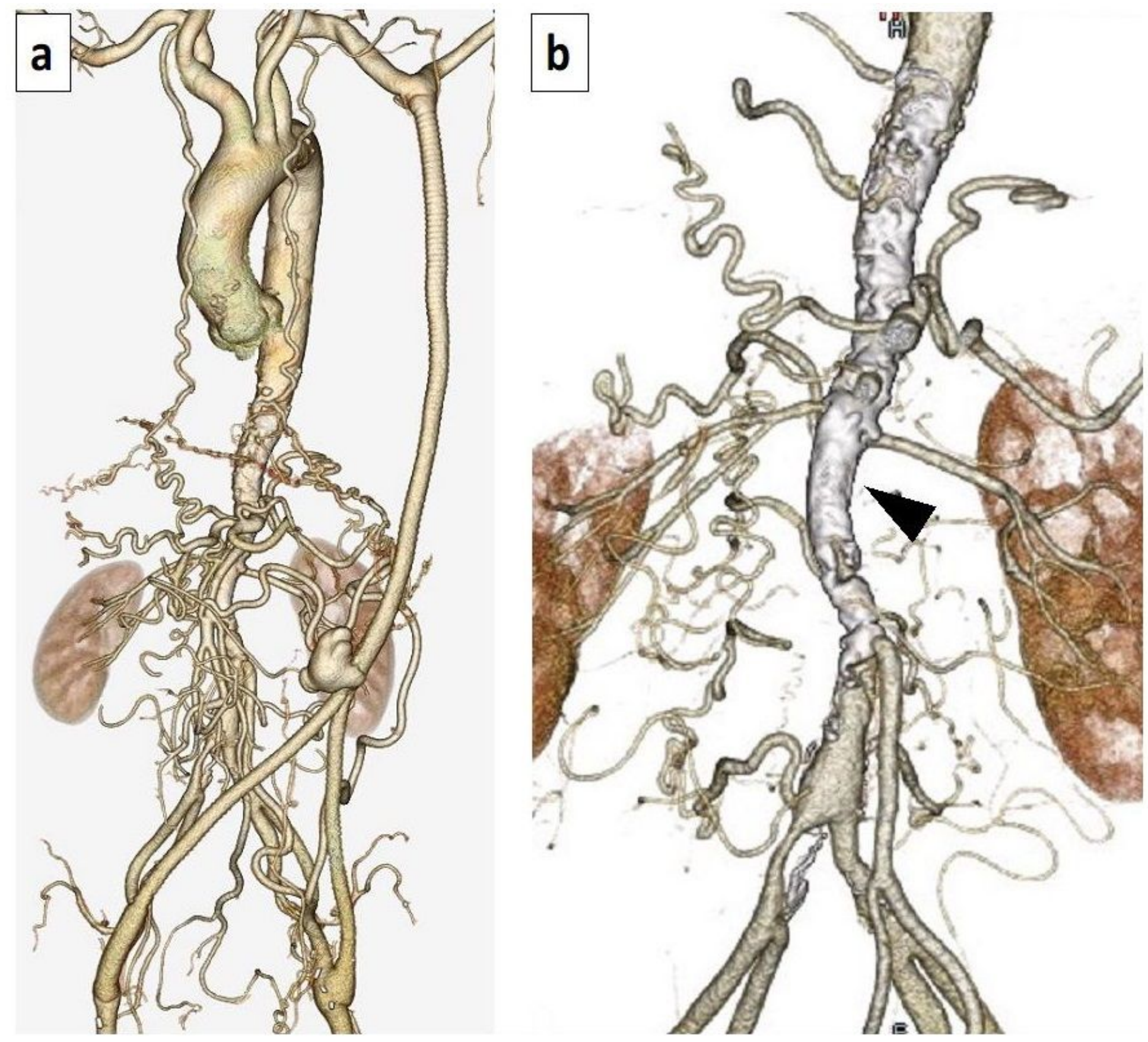

Figure 1

Preoperative computed tomography (CT) a: Preoperative 3D-CT shows a patent left axilobifemoral bypass. Many collateral circulations to the lower extremity arteries are present b: 3D-CT of the thoracoabdominal aorta shows a high degree of calcification and stenosis around the descending aorta to the abdominal aorta (arrowhead). 

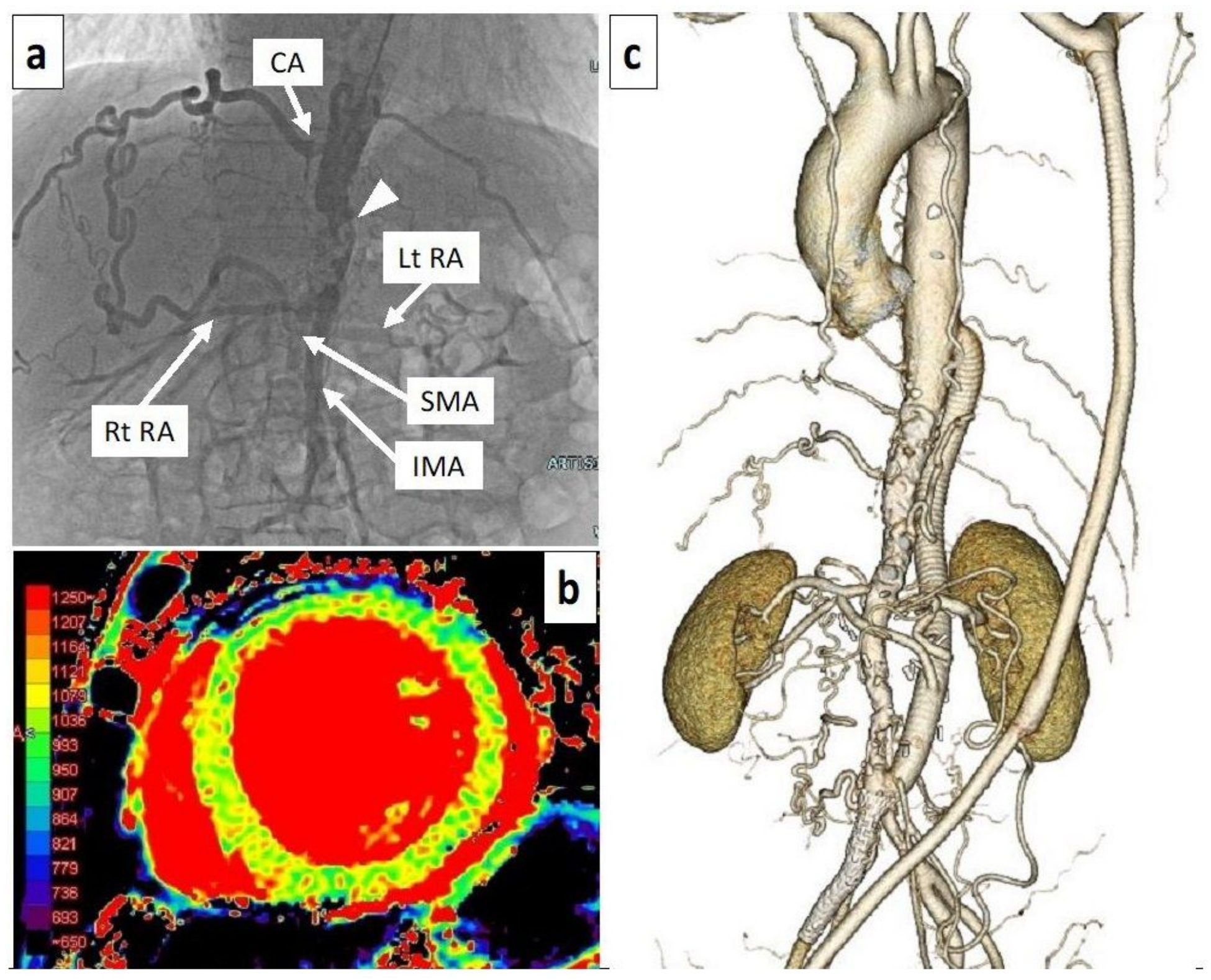

Figure 2

Preoperative aortography, magnetic resonance imaging (MRI), and postoperative computed tomography (CT) a: Aortography shows the superior mesenteric artery imaged by the developed collateral circulation from the celiac artery (CA). Bilateral renal arteriography was delayed. Severe stenosis is observed in the epigastric aorta of the renal artery (arrowhead). b: T1 mapping images on visceral MRI shows a slight increase in the T1 value of the left ventricular wall and no fibrosis progression on the left ventricular wall. c: Postoperative 3D-CT shows that the descending thoracic aorta-abdominal aorta bypass and bilateral renal artery bypass were patent. 

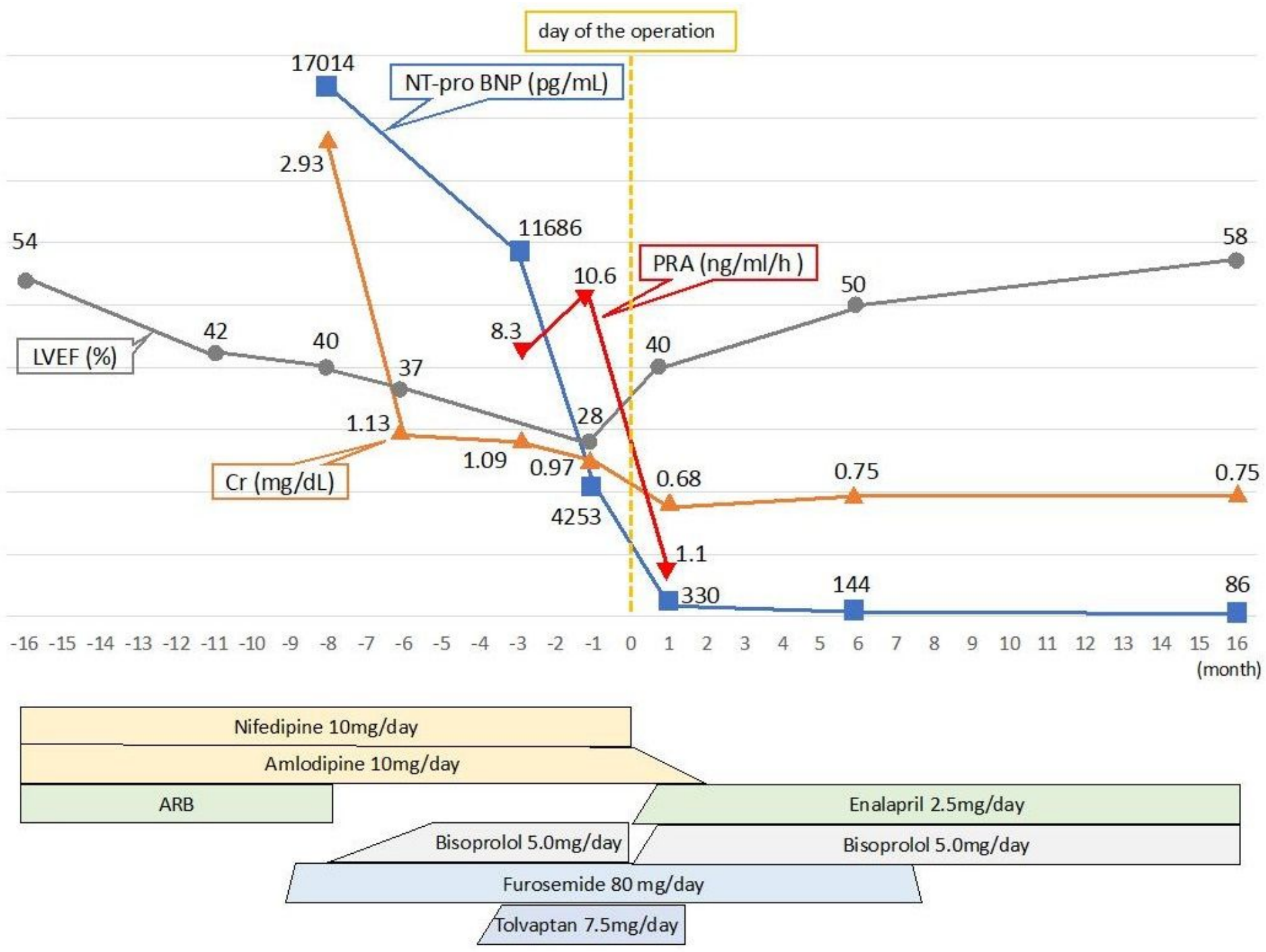

Figure 3

Pre- and postoperative progress 0 month indicates the month of the surgery. Six months postoperatively, cardiac function improved to preoperative levels. NT-proBNP and Cr levels appeared to decrease with the control of heart failure, but a further decrease is observed postoperatively. PRA is high and tends to increase preoperatively, but decreases postoperatively. The dose of antihypertensive drugs can be reduced postoperatively. In addition, diuretics used to control heart failure can be discontinued postoperatively ARB, angiotensin II receptor blocker; $\mathrm{Cr}$, creatinine; LVEF, left ventricular ejection fraction; NT-proBNP, N-terminal pro-brain natriuretic peptide; PRA, plasma renin activity

\section{Supplementary Files}

This is a list of supplementary files associated with this preprint. Click to download.

- CAREchecklistEnglish.pdf 